\title{
Interactive comment on "The potential of urban rainfall monitoring with crowdsourced automatic weather stations in Amsterdam" by L. de Vos et al.
}

\section{Anonymous Referee \#2}

Received and published: 15 November 2016

\section{Summary}

This study discusses a new technique for measuring rainfall. This consequently would satisfy the need for hydrological analyses like urban hydrology, where high temporal and spatial resolution rainfall data are required. Although several studies have addressed alternative ways for measuring rainfall such as using microwave links, this study specifically investigates rainfall information from automatic personal weather stations (PWSs) for point-measurement purposes. I found the topic quite interesting as such approaches may indeed provide valuable information.

General comments:

I found the manuscript a bit hard to follow. The authors may consider the following points for improvements. The structure of the manuscript may need some modifica- 
tions. For example, you may introduce the "Methodology" the "Data and study area" in separated sections, and not combined. Providing some important details would make getting the message of the manuscript easier. More information is required explaining the functionality of the devices used in this study. Although some devices do not have the sought information, providing available information, e.g. for Netatmo, would help to better follow the text. You may even add some figures for that reason. The message of some sentences is not clear. Furthermore, some sentences have minor/major language problems and/or they are too long. You may consider English proofreading. Another point is the objective of this study. The last paragraph in introduction addresses the objective of this study. You may clearly add the fact that using these measurements is economically reasonable comparing to conventional techniques. At the end I would like to add that there are some facts given without proper references.

Scientific comments:

You addressed a study where they used "average value of 12 pixels", and furthermore, you addressed the difficulties radar data have, especially when comparing to "groundbased measurements". You used in this study 1 radar pixel as the reference at the end. You may elucidate the reason for that. Also, you may explain the method (you) used for correcting (adjusting) radar data.

The same is valid for the experimental set-up. You may add how exactly the reference device "KNMI pit gauge" measures rainfall.

I could not really understand the reason why you used Pearson correlation coefficient for your analyses. You may add some other criteria like bias, root mean square error, etc. Regarding the "inter-station correlation", you may add the way you estimated the parameters.

Printer-friendly version

Specific comments:

You may address the specific comments by considering the points mentioned above.

Discussion paper 
Some of the specific comments for the abstract and introduction section is provided in the following:

P.1 - L.7: are you referring by "63 stations" to the Netatmo network in Amsterdam?

P.1 - L.10: "the method of data transfer to the online platform causes considerable errors in the datasets obtained." This phrase may need some modifications.

Interactive

comment

P.1 - L.16: Does "data conversion" refer to "data transfer" you provided some lines earlier? The word "conversion" sounds a bit strange in this contest.

P.2 - L.3: Why "Rainfall" starts with capital letter? And "from the radar"; you may change to "from the radar station".

P.2 - L.4: ". . and which may.." I suppose one may not put "and" and "which" together.

P.2 - L.7: Does "spatial resolution" mean "network density"?

P.2 - L.10: You may add a comma after "Hydrological models" and a comma before "have minimized".

P.2 - L.15: What is the spatial resolution of $3 \mathrm{~km}$ ? Is it a $3 \mathrm{~km} \times 3 \mathrm{~km}$ ?

P.2 - L.21: Bruni et al. (2015) "addressed" ....

P.2 - L.24: When explaining "Smearing"; “...the ratio of the resolution...": are you referring to temporal, spatial or tempo-spatial resolution?

P.2 - L.30: Ochoa-Rodriguez et al. (2015) "evaluated the ..."

P.2 - L.31: You may add a comma after "1 km" and a comma before "was found".

P.2 - L.34: Not all radar products are in $5 \mathrm{~min}$ temporal resolutions. Some X-Band radars work in 30-sec temporal resolution.

P.2 - L.35: "... impact on the accuracy than coarsening spatial scales does." I would change the word scale to "resolution". Furthermore, you may omit "does" at the end of

Printer-friendly version

Discussion paper 
the sentence.

P.3 - L.2: “. . . evaluate” to ". . . evaluated”. You may rephrase the entire sentence.

P.3 - L.9: What type of sensors are you referring to? Rain gauges?

P.4 - L.10: But you did not investigate for any urban applications. Your study area is located in an urban area.

Interactive

comment

Interactive comment on Hydrol. Earth Syst. Sci. Discuss., doi:10.5194/hess-2016-505, 2016. 